\title{
Rechtsgeschichte
}

www.rg.mpg-de

http://www.rg-rechtsgeschichte.de/rg14

Zitiervorschlag: Rechtsgeschichte Rg 14 (2009)

Rg 142009

$148-167$

http://dx.doi.org/10.12946/rg14/148-167

\section{Christoph Safferling}

\section{Lernen von Nürnberg}

Die Relevanz des Nürnberger Hauptkriegsverbrecherprozesses für das moderne Völkerstrafrecht 


\section{Abstract}

The significance of the trial against the major Nazi war criminals before the International Military Tribunal (IMT) at Nuremberg can be shown in four different ways: (1) impunity for heads of state and other members of the military or political elite of a state does not apply to a set of core international crimes. (2) There exist a number of crimes which are universally accepted as international crimes, which are aggression, war crimes and crimes against humanity. The crime of genocide was added to the list of these Nurembergcrimes by the so-called genocide convention of 1948. (3) The attribution of guilt follows a set of general principles of criminal law as e. g. the mens rea-principle. In general »superior order« cannot operate as an excuse; similarly a military or political commander can be held responsible for the conduct of his subordinate. A rather difficult and contested issue is the attribution of guilt by means of "conspiracy« as was foreseen in the Charter of the IMT. Modern international criminal law follows a similar concept, which is called "joint criminal enterprise «, as is found in the jurisprudence of the International Criminal Tribunal for the former Yugoslavia. The crucial issue in attributing guilt thereby lies in the »common plan«, whereas the actual conduct is of minor relevance. (4) Finally, the Nuremberg proceedings have shown that in principle a criminal trial must be fair and must adhere to the presumption of innocence.

However, it is difficult to prove whether a criminal trial can add to reconciliation and to preventing future crimes. Although it is fair to say that Nuremberg was the beginning of a prosperous time for Europe, criminal prosecution is not always an adequate way to help societies in transition. 


\section{Lernen von Nürnberg}

Die Relevanz des Nürnberger Hauptkriegsverbrecherprozesses für das moderne Völkerstrafrecht

I. Der Nürnberger Prozess als gemeinsame europäische Rechtserfahrung

Spricht man in Europa von Freiheit und Rechtsstaatlichkeit, nimmt Nürnberg eine bedeutsame Rolle ein. Die Reichsparteitage ${ }^{\mathrm{I}}$ ebenso wie die sog. Nürnberger Rassengesetze ${ }^{2}$ stehen als Symbol für Unterdrückung und Tyrannei; der Schwurgerichtssaal des Landgerichts Nürnberg-Fürth, in dem I945/46 der Nürnberger Prozess abgehalten wurde, symbolisiert Bestrafung und Wiedergutmachung im Falle schwerwiegendster Verbrechen gegen die Menschlichkeit. Europa hat nach dem Ende des Zweiten Weltkrieges einen Prozess der Vereinigung und Versöhnung eingeläutet, der in den letzten 60 Jahren zu einer ungewohnt friedlichen und prosperierenden Zeit geführt hat. Im Prozess des Erinnerns an die Geschichte Europas und in dem gleichzeitigen Bemühen, die Verbrechen der Vergangenheit nicht zu wiederholen, ist der Nürnberger Prozess von Wichtigkeit. Er bezeichnet einen Wendepunkt in der Menschheitsgeschichte, da zum ersten Mal die höchsten Staatsmänner, die politischen Führer und die militärische Elite wegen ihrer im Amt begangenen Taten wie »gewöhnliche "Verbrecher vor einer Richterbank zur Rechenschaft gezogen und bestraft wurden. ${ }^{3}$

Diese sehr symbolträchtige und medienwirksame Aufarbeitung der nationalsozialistischen Aggression mit den Mitteln des Strafrechts auf internationaler Ebene wirkte in ihrem strafrechtlichen Kern lange Zeit nur verdeckt nach. Erst fast fünfzig Jahre nach der Urteilsverkündung in Nürnberg am 30. September und I. Oktober I 946 wurde am 27. Mai I 993 durch den Sicherheitsrat der Vereinten Nationen ein Ad-hoc-Tribunal zur Verfolgung der Verbrechen, die auf dem Gebiet des ehemaligen Jugoslawiens begangen wurden, eingerichtet. ${ }^{4}$ Am I 7. Juli I 998 wurde ein Statut für einen permanenten Internationalen Strafgerichtshof (IStGHSt) in Rom verabschiedet, ${ }^{5}$ das am I. Juli 2002 in Kraft getreten ist. Der Nürnberger Prozess dient für diese Entwicklungen als Vorbild. Die Verweise auf diese Vorbildfunktion sind allerdings häufig pauschal und eher politischer Natur. Deshalb sei die Frage erlaubt,

I Vgl. dazu auch Egon Fein, Hitlers Weg nach Nürnberg: Verführer.
Täuscher. Massenmörder, Nürnberg 2002.

2 »Gesetz über das Reichsbürgerrecht « und "Gesetz zum Schutze des deutschen Blutes und der deutschen Ehre« verabschiedet auf einer Sondersitzung des Reichstags in Nürnberg anlässlich des Reichsparteitags am I 5 . September I935; vgl. dazu KLAUS KastNeR, Rückblick: NSDAP - Reichsparteitage - Rassengesetze, in: Die
Nürnberger Prozesse. Völkerstrafrecht seit I945, 2006, hg. von Herbert Reginbogin und Christoph SAFFerling, München 2006, II4.

3 Whitney Harris, in: Die Nürnberger Prozesse. Völkerstrafrecht seit I945 (Fn. 2) IOO-IO5.

4 Auf der Grundlage von UN Sicherheitsratsresolution 827 (I993).

5 Sog. Römisches Statut, UN Doc.A/CONF.I $83 / 9$. 
welche Signifikanz Nürnberg für das moderne Völkerstrafrecht tatsächlich hat und was es heißt, von Nürnberg zu lernen.

Dabei sollen vier einzelne Lernerfahrungen unterschieden werden: (I) die Idee einer internationalen Strafjustiz als über dem Grundsatz der Staatssouveränität stehend, (2) die Entwicklung allgemein akzeptierter internationaler Kernverbrechen, (3) die Anwendung anerkannter strafrechtlicher Zurechnungsprinzipien als »Allgemeiner Teil « eines Völkerstrafrechts, und (4) das Fair-trialPrinzip als Grundlage eines rechtsstaatlichen Strafverfahrens.

\section{Einzelne Lernerfahrungen}

I. Durchbrechung der Staatssouveränität

a) Internationale und nationale Durchsetzung von Völkerstrafrecht

Oft wird im Plural von den Nürnberger Prozessen gesprochen. ${ }^{6}$ Angesichts der Frage der Durchsetzungsebene muss allerdings unterschieden werden: Im Nürnberger Schwurgerichtssaal wurden zwei phänotypisch verschiedene Prozessarten durchgeführt. Zum einen der sog. Nürnberger Hauptkriegsverbrecherprozess, der basierend auf der am 8. August 1945 in London verabschiedeten Charta des internationalen Militärgerichtshofs (IMT-Statut) ein wirklich internationales Unterfangen war. ${ }^{7}$ Auf der Grundlage dieser Charta wurden von den alliierten Siegermächten, den USA, dem Vereinigten Königreich, Frankreich und der Sowjetunion, 2 I vormalige NS-Größen verfolgt. ${ }^{8}$ Das Statut wurde von I8 weiteren Staaten unterzeichnet und war daher getragen von einer breiten Unterstützung. ${ }^{9}$ Entgegen ursprünglichen Planungen fanden in der Folge keine weiteren wirklich internationalen Strafverfahren mehr statt. ${ }^{\text {IO }}$ Der sog. Kalte Krieg war bei Beendigung des Hauptkriegsverbrecherprozesses am I. Oktober 1946 bereits so weit fortgeschritten, dass kein zweites Mal eine Kooperation zwischen den westlichen Siegermächten und der Sowjetunion vorstellbar war. ${ }^{\text {II }}$ Weitere NS-Verbrecher wurden von nationalen Gerichten verfolgt und abgeurteilt. Die Verfahren, die als »Nürnberger Nachfolgeprozesse « in die Geschichte eingegangen sind, waren Prozesse, die auf der Grundlage des Kontrollratsgesetzes $\mathrm{Nr}$. Io allein in US-amerikanischer Verantwortung standen. ${ }^{\mathbf{2}}$ Parallel wurden sowohl in der französischen ${ }^{\mathbf{1 3}}$ wie der

6 In Tokio wurde ein vergleichbarer Prozess gegen japanische Kriegsverbrecher durchgeführt. Dieser fand allerdings keine vergleichbare Resonanz; vgl. dazu etwa PHILIPP Osten, Der Tokioter Kriegsverbrecherprozess und die japanische Rechtswissenschaft, Berlin 2003.

7 Abgedruckt in: Internationaler Militärgerichtshof, Amtliche Sammlung, Nürnberg I947, Band I, 7 ff.
8 Von den ursprünglich 24 Angeklagten wurde gegen Gustav Krupp von Bohlen und Halbach das Verfahren wegen Verhandlungsunfähigkeit eingestellt, der Angeklagte Robert Ley konnte sich dem Verfahren durch Suizid entziehen und gegen Martin Bormann wurde in Abwesenheit verhandelt, was nach Art. I 2 des IMT-Statuts gestattet war.
9 Das waren: Griechenland, Dänemark, Jugoslawien, die Niederlande, Tschechoslowakei, Polen, Belgien, Äthiopien, Australien, Honduras, Norwegen, Panama, Luxemburg, Haiti, Neuseeland, Indien, Venezuela, Uruguay und Paraguay.

IO Vgl. Art. 22 IMT-Statut.

i I Vgl. Klaus Kastner, Von den Siegern zur Rechenschaft gezogen, Nürnberg 200I, 35 .

I 2 Weitere Verfahren wurden unter US-amerikanischer Verantwortung in Dachau durchgeführt. Diese waren allerdings Verfahren vor Militärkommissionen. Vgl. Holger Lessing, Der erste Dachauer Prozess (I945/46), Baden-Baden I993; Michael Bryant, Dachau Trials - Die rechtlichen und historischen Grundlagen der US-amerikanischen Kriegsverbrecherprozesse, I942-I947, in: Historische Dimensionen von Kriegsverbrecherprozessen nach dem Zweiten Weltkrieg, hg. von HeNNING Radtke, Dieter Rössner, Theo SCHILler und Wolfgang Form, Baden-Baden 2007, I I I-I 22, und den Sammelband Dachauer Prozesse. NS-Verbrechen vor amerikanischen Militärgerichten in Dachau I945-I948, hg. von Ludwig EIber und Robert SIGEL, Göttingen 2007.

I 3 Vgl. Claudia Moisel, Frankreich und die deutschen Kriegsverbrecher, Göttingen 2004. Die Verfahren sind bislang wenig aufgearbeitet. 
I4 Vgl. zu den Verfahren unter dem Royal Warrant vom I8. Juni I945: KatRin Hassel, Kriegsverbrechen vor Gericht, Baden-Baden 2009.

I 5 Vgl. HinRich RüPING, Zwischen Recht und Politik: Die Ahndung von NS-Taten in beiden deutschen Staaten nach 1945 , in: Die Nürnberger Prozesse. Völkerstrafrecht seit I945 (Fn. 2) I 86-I95; ANNETte Weinke, Die Nürnberger Prozesse, München 2006, I I 2II 5 .

I6 Einen Überblick bieten: KASTNER (Fn. II) 227-283; WHITNEY HARRIS, Tyrannen vor Gericht, Berlin 2008, 532-550.

I7 Dies ist der bislang einzig vollständig dokumentierte Nachfolgeprozess, vgl. Angelika Ebbinghaus, KLAus DöRner u. a., Der Nürnberger Ärzteprozess I946/47. Wortprotokolle, Anklage- und Verteidigungsmaterial, München I999.

I 8 Dazu der Sammelband: Das Nürnberger Juristen-Urteil von 1947 , hg. von Lore Peschel-Gutzeit, Baden-Baden 1996.

I9 Speziell zu Fall V, dem Prozess gegen Flick u. a.: SUSANNE JUNG, Die Rechtsprobleme der Nürnberger Prozesse, Tübingen 1992.

\% 20 Vgl. Rainer S. Blasius, Fall I I:
Der Wilhelmstraßen-Prozess, in:

britischen, ${ }^{\mathbf{I} 4}$ aber auch in der sowjetisch besetzten Zone ${ }^{\mathrm{I} 5}$ Verfahren gegen Kriegsverbrecher abgehalten, die allerdings nicht die gleiche Aufmerksamkeit und Bedeutung erlangten wie die in Nürnberg bis 1949 durchgeführten zwölf Nachfolgeprozesse. ${ }^{\text {I }}$ Das lag auch daran, dass die Anklagestrategie der Vereinigten Staaten eine Konzentration auf verschiedene Berufsgruppen zum Inhalt hatte. Es wurden also gleichsam Stellvertreter-Prozesse geführt gegen Ärzte, ${ }^{17}$ Juristen, ${ }^{\text {I8 }}$ Mitglieder von Einsatzgruppen, gegen Industrielle $^{19}$ und Diplomaten. ${ }^{20}$ Während also der Hauptkriegsverbrecherprozess für die internationale Strafjustiz als Vorbild, in eingeschränktem Sinn als Präzedenz, gelten darf, stellen die Nachfolgeprozesse keine Form der internationalen Durchsetzung von Völkerstrafrecht dar. Allerdings bleiben sie trotz ihres nationalen Charakters für die Entwicklung des materiellen Völkerstrafrechts und für den moralischen Strafanspruch der internationalen Gemeinschaft bedeutsam.

b) Immunität vs. Strafanspruch

(I) Leipziger Prozesse

Im Nürnberger Hauptkriegsverbrecherprozess wurden Grundprinzipien der westfälischen Völkerrechtsordnung wie die Staatssouveränität und der Grundsatz der Immunität von Staatsoberhäuptern und Regierungsmitgliedern sowie das Rückwirkungsverbot als Säule eines aufgeklärten, demokratischen Strafrechts zugunsten einer Bestrafung führender Staatsmänner zurückgestellt. Bereits nach dem Ersten Weltkrieg war eine Bestrafung des als Aggressor ausgemachten deutschen Kaisers Wilhelm II. im Versailler Vertrag vorgesehen (Art. 227 Versailler Vertrag). Allerdings kam es vor allem aus zwei Gründen nie zu einem solchen Verfahren: (I) Der auf einem britischen Vorschlag basierende Straftatbestand, Verbrechen gegen die »Heiligkeit der Verträge ", war reichlich unbestimmt und damit unanwendbar. ${ }^{2 \mathrm{I}}$ (2) Die Niederlande gewährten dem verfolgten Kaiser nach einer längeren Auseinandersetzung politisches Asyl. ${ }^{22}$ Prozesse gegen Kriegsverbrecher durchzuführen, wurde auf der Grundlage des Versailler Vertrages schließlich Deutschland, also der nationalen Justiz, anheim gestellt. ${ }^{23}$ Die alliierten Siegermächte behielten sich aber das Recht vor, die Verfahren vor eigenen Gerichten zu wiederholen, sollte die Strafverfolgung in Deutschland nicht befriedigend ausfallen. ${ }^{24}$ Aus der

Der Nationalsozialismus vor Gericht, hg. von GERD UEBERSCHÄr, 2. Aufl., Frankfurt a. M. 2000, I90.

2 I Vgl. Cherif M. Bassiouni, World War I: » The War to End all Wars « and the Birth of a Handicapped International Criminal Justice System, in: Denver Journal of International Law and Policy 30 (2002) 244-296, 27I-275. 22 Vgl. Bassiouni (Fn. 2I) 283-285.
23 Nach Art. 228 des Versailler Vertrages stellten die Alliierten Regierungen eine Namensliste mit vermeintlichen deutschen Kriegsverbrechern zusammen. Vorgesehen war zunächst eine Auslieferung dieser Personen, was die deutsche Regierung durch die Verfolgung von Kriegsverbrechen durch eigene Gerichte abzuwenden wusste.

24 Vgl. Bassiouni (Fn. 2I) 286. 
langen Liste von ca. 890 vermeintlichen Kriegsverbrechern wurden schließlich in den Jahren I92 I/22 zwölf Personen vor dem Reichsgericht angeklagt. Diese Verfahren sind als sog. Leipziger Prozesse in die Geschichte eingegangen. ${ }^{25}$ Die geringe Zahl der tatsächlich Verfolgten wurde begleitet von der großen richterlichen Milde; schließlich kam es zu acht Verurteilungen. ${ }^{26}$ Eines haben diese Verfahren deutlich gemacht: Die nationale Justiz ist nicht uneingeschränkt geeignet, eigene Staatsangehörige als Kriegsverbrecher zu verfolgen. Die milden Haftstrafen verbunden mit vorzeitigen Entlassungen und Begnadigungen sprechen von einem Misserfolg auf ganzer Linie, legt man einen gewissen Abschreckungseffekt von Strafprozessen als Maßstab an die Leipziger Prozesse an. ${ }^{27}$

\section{(2) Der Nürnberger Prozess}

Nach früheren Anzeichen ${ }^{28}$ legten sich die Alliierten im Zweiten Weltkrieg spätestens in der Moskauer Deklaration vom 30. Oktober I943 auf eine Strafverfolgung der schlimmsten NS-Verbrecher nach dem Sieg über Nazi-Deutschland fest. ${ }^{29}$ Die Strafverfolgung sollte auf verschiedenen Ebenen stattfinden, und insbesondere dort, wo es keinen expliziten territorialen Bezug gab, sollte eine koordinierte internationale Strafverfolgung durchgeführt werden. ${ }^{3 \circ}$ Nach der bedingungslosen Kapitulation des Deutschen Reichs am 8. Mai 1945 begann daher neben der verwaltungstechnischen Organisation der Besatzungszonen zugleich die Arbeit an einer internationalen Strafverfolgung der Hauptkriegsverbrecher. Auch wenn sich mit Hitler und Goebbels die bedeutendsten Repräsentanten des NS-Regimes bereits durch Selbstmord der Strafverfolgung entzogen hatten, standen insbesondere Hermann Göring, Rudolf Heß als Hitlers Stellvertreter, Karl Dönitz als Hitlers Nachfolger, mehrere Wehrmachtsgeneräle wie Wilhelm Keitel und Alfred Jodl, verschiedene Reichsminister wie Joachim von Ribbentrop und Albert Speer, sowie weitere Repräsentanten des Unrechtssystems, unter diesen auch der Nürnberger Judenhetzer Julius Streicher, zur Verfügung. Mit der Anklage der politischen Führungsebene des NS-Regimes wird klar, dass der Grundsatz der Immunität für Taten, die in Ausübung eines öffentlichen Amtes begangen werden (»Act of state«-doctrine), bei schweren Menschlichkeitsverbrechen keine Gültigkeit mehr hat. Die Anklage der obersten Militärs verdeutlicht, dass eine Berufung auf »Handeln auf Befehl« keinen Ausschlussgrund für die individuelle Ver-

25 Gerd Hankel, Die Leipziger Prozesse, Hamburg 2003; HARALD WiggenHORN, Verliererjustiz. Die Leipziger Kriegsverbrecherprozesse nach dem Ersten Weltkrieg, Baden-Baden 2005.

26 Davon wurden aber nur vier wegen völkerrechtlicher Verbrechen im eigentlichen Sinne verfolgt; vgl. Katrin Hassel, Kriegsverbrechen vor Gericht, Baden-Baden 2009, 55 .
27 Dieser Zusammenhang wird von Robert H. JACKSON, dem USamerikanischen Chefankläger in Nürnberg in seinem Eröffnungsplädoyer deutlich ausgesprochen, vgl. Internationaler Militärgerichtshof, Band II, Ioo; vgl. auch JUNG (Fn. I9) 96-I06.

28 Bereits am 25. Oktober I94I erklärte Churchill die Bestrafung der NS-Führer zum Kriegsziel, vgl. Internationaler Militärgerichtshof,
Amtliche Sammlung, Nürnberg I947, Band V, 464; am I3. Januar I 942 folgte die sog. Erklärung von St. James der in London befindlichen Exilregierungen, in welcher eine gerichtliche Bestrafung der Kriegsverbrecher gefordert wurde. 29 HARris (Fn. I6) 5 f.; KASTNER (Fn. II) $25 \mathrm{f}$.

30 Der letzte Satz der Deklaration lautet: "The above declaration is without prejudice to the case of German criminals whose offenses have no particular geographical localization and who will be punished by joint decision of the government of the Allies. « 
3I Beide Ausschlussgründe werden von Robert H. Jackson in seinem Eröffnungsplädoyer als unzeitgemäß und dem Grundsatz individueller Verantwortlichkeit widersprechend bezeichnet, vgl. Internationaler Militärgerichtshof, Amtliche Sammlung, Nürnberg I947, Band 2, I49.

32 Ralph Percy, Realms of Value, Cambridge/Mass. I945, $255 \mathrm{f}$.

33 Vgl. Harris (Fn. I6) $502 \mathrm{f}$.

34 UN GA Resolution 95 (I) vom I I. Dezember 1946. Der Rechtsausschuss wurde mit einer Ausarbeitung der "Nürnberg Principles « beauftragt. Diese Arbeiten wurden im Jahr I950 abgeschlossen und der Generalversammlung vorgelegt, vgl. Yearbook of the International Law Commission, I950, Band II, [curr] 97.

35 Kаг Амвоs, Internationales Strafrecht, 2. Aufl., München 2008, \IO Rn. 67.

36 Vgl. Jens Meyer-Ladewig, EMRK-Handkommentar, 2. Auf. Baden-Baden 2006, Art. 7 Rn. I I f.

37 Siehe 6. Bericht der Bundesregierung über ihre Menschenrechtspolitik in den auswärtigen Angelegenheiten und in anderen

antwortlichkeit bietet. ${ }^{3 \mathbf{I}}$ Das gilt selbst dann, wenn das jeweilige nationale Recht so ausgestaltet ist, dass die Taten keinen Gesetzesverstoß darstellen. Auch wenn eine rückwärtige Anwendung von Strafrecht nach allgemeinen rechtsstaatlichen Prinzipien nicht statthaft ist, so kann das doch dort nicht gelten, wo die Grundlagen der zivilisierten Welt selbst von den angeklagten Personen angegriffen wurden. ${ }^{32}$ Diese einer streng positivistischen Rechtsdogmatik zunächst widersprechenden Grundannahmen basieren auf einer zutiefst moralischen Überzeugung, darüber hinaus aber auch auf einem allgemeinen Gerechtigkeitsverständnis. ${ }^{33}$ Kurz nach Abschluss der internationalen Strafverfolgung von NS-Verbrechen in Nürnberg hat nicht nur die Generalversammlung der Vereinten Nationen die »Nürnberg Principles « verabschiedet und damit den Grundsatz der individuellen Verantwortlichkeit von Staatenführern jenseits der Staatssouveränität anerkannt; ${ }^{34}$ auch die europäischen Staaten haben sich mit der Verabschiedung der Europäischen Menschenrechtskonvention (EMRK) und der Gründung des Europarates eine menschenrechtliche Verfassung gegeben, die diese Werte widerspiegelt. So wurde in Art. 7 EMRK der Grundsatz des Rückwirkungsverbotes anerkannt, zugleich in dessen Abs. 2 aber festgestellt, dass dieses Rückwirkungsverbot dann keine Wirkung haben kann, wenn die Tat nach den von den zivilisierten Völkern anerkannten allgemeinen Rechtsgrundsätzen strafbar war. Diese Ausnahme vom nullum crimen-Grundsatz wurde zunächst von der Bundesrepublik Deutschland als offensichtlicher Versuch, die Nürnberger Prozesse im Nachhinein zu rechtfertigen (sog. Nürnberg Klausel), ${ }^{35}$ bei der Ratifizierung der EMRK mit einem Vorbehalt versehen. ${ }^{36}$ Nach dem Wiederaufleben der Idee einer internationalen Strafjustiz zunächst durch die Schaffung des Jugoslawientribunals im Jahr I993, sodann durch die Verabschiedung und Errichtung des Internationalen Strafgerichtshofs, ist die Bundesrepublik Deutschland im Jahre 200 I auch formal auf die europäische Linie eingeschwenkt und hat den Vorbehalt zu Art. 7 Abs. 2 EMRK offiziell zurückgezogen. ${ }^{37}$

\section{(3) Nach Nürnberg}

Die Legitimität von Strafverfahren hängt allerdings auch von einer gleichmäßigen Anwendung ab. Eine selektive oder gar willkürliche Bestrafung widerspricht elementar dem Rechtsstaatsprinzip. Nach Nürnberg sah sich das Völkerstrafrecht in genau dieser

Politikbereichen (Human Rights Report), 2000/02, 36 unter: www.bmj.bund.de/media/archive/ 267.pdf. Die Rücknahme der Reservierung war auch deshalb erforderlich geworden, da nach der Herstellung der deutschen Einheit die Frage des Rückwirkungsverbots erneut aktuell wurde. Das zeigte insbesondere der Fall vor dem EGMR Streletz, Kess ler, Krenz gegen Deutschland,
22. März 200I, Reports 200I-II; zuvor BVerfGE 95, 96; dazu auch Gerhard Werle, Rückwirkungsverbot und Staatskriminalität, in: NJW 200I, 300I; Anne Peters, Einführung in die EMRK, München 2003, 6. Im größeren $\mathrm{Zu}$ sammenhang auch Claus Kress, Versailles - Nürnberg - Den Haag: Deutschland und das Völkerstrafrecht, in: JZ 2006, 98I99I. 
Falle, die der amerikanische Chefankläger und große Visionär Justice Robert H. Jackson in seinem Eröffnungsplädoyer in Nürnberg bereits beschwor: ${ }^{38}$

»Und lassen Sie es mich deutlich aussprechen: Dieses Gesetz wird hier zwar zunächst auf deutsche Angreifer angewandt, es schließt aber ein und muß, wenn es von Nutzen sein soll, den Angriff jeder anderen Nation verdammen, nicht ausgenommen die, die jetzt hier zu Gericht sitzen. « 39

Der Kalte Krieg machte es politisch unmöglich, die Versuche, die seitens der Vereinten Nationen unternommen wurden, einen permanenten Strafgerichtshof einzurichten, in die Tat umzusetzen. Bemühungen dieser Art wurden offiziell bereits in den späten Fünfzigerjahren wieder eingestellt. ${ }^{4}$ Auf anderen Ebenen jedoch wirkte das in Nürnberg Erreichte fort. So wurden die Genfer Konventionen bereits am I 2. August I 949 neu formuliert und jedenfalls für den internationalen bewaffneten Konflikt mit einer ganzen Reihe an konkreten strafrechtlichen Drohungen versehen. Am I 8. Juni 1977 wurde in den Zusatzprotokollen ein weiterer Fortschritt erreicht. Ähnliches entwickelte sich auch auf der Ebene der Menschenrechte. Nicht nur im Zusammenhang mit dem Europarat und der EMRK wurden hier neue Maßstäbe gesetzt, auch auf globaler Ebene wurde 1948 durch die Allgemeine Erklärung der Menschenrechte und I966 mit der Verabschiedung des internationalen Pakts für bürgerliche und politische Rechte ein menschenrechtlicher Durchbruch gefeiert. All das sind Nachwirkungen von Nürnberg. Eine internationale Strafverfolgung fand indes nicht mehr statt, auch wenn es an Gelegenheiten nicht gemangelt hat.

Die Legitimität von Nürnberg war fast $5 \circ$ Jahre lang mit dem Makel behaftet, dass die einzigen Verfolgten die besiegten Deutschen waren. In Deutschland war deshalb die Meinung weit verbreitet, die Nürnberger Prozesse wären ein Akt von »Siegerjustiz « gewesen. ${ }^{4 \mathrm{I}}$ Ein Durchbruch gelang erst nach dem Ende des Kalten Krieges. Die Vereinten Nationen reagierten auf den mit kriegerischen Mitteln durchgeführten Zerfall des Vielvölkerstaates Jugoslawien mit der Errichtung eines internationalen Straftribunals als Unterorgan des Sicherheitsrats der Vereinten Nationen. ${ }^{42}$ So ungewöhnlich dieser Schritt formal wie politisch war, so sehr zeugte er doch davon, dass die Idee einer internationalen Strafverfolgung, wie sie in Nürnberg praktiziert wurde, noch lange nicht in Vergessenheit geraten war. Eher im Gegenteil: Das Statut des Jugosla-

38 Zur Rolle Jacksons in Nürnberg, vgl. John BARretT, »One Good Man « - The Jacksonian Shape of Nuremberg, in: Die Nürnberger Prozesse. Völkerstrafrecht seit I945 (Fn. 2) I 2O-I 29.

39 Justice Robert H. Jackson im Eröffnungsplädoyer am 2I.I I.I945, Internationaler Militärgerichtshof, Amtlicher Text, Nürnberg 1947, Band 2, I 82.
40 Der Draft Code of Crimes against Peace and Security of Mankind des Rechtsausschusses der Vereinten Nationen, Yearbook of the International Law Commission, I954, Band II, UN Doc. A/2693, wurde in der Generalversammlung zwar beraten, es sollte aber zunächst eine Definition der Aggression erarbeitet werden, bevor eine umfassende Kodifikation des Völkerstrafrechts wieder auf die
Tagesordnung gesetzt werden sollte; vgl. GA Res. I 68 (XII) vom II. Dezember 1957.

4I Dazu Albin Eser, Das Internationale Militärtribunal von Nürnberg aus deutscher Perspektive, in: Die Nürnberger Prozesse. Völkerstrafrecht seit I945 (Fn. 2) 53-59. 42 UN Sicherheitsratsresolution 827 (I993); vgl. dazu Herwig RoggeMANN, Der Internationale Strafgerichtshof der Vereinten Nationen von 1993 und der Krieg auf dem Balkan, Berlin I994. 
43 Der damalige Generalsekretär der Vereinten Nationen Butros Butros-Ghali nahm in seinem vorbereitenden Bericht zur Errichtung des Jugoslawientribunals mehrmals ausdrücklich Bezug auf den Internationalen Militärgerichtshof in Nürnberg, vgl. Report of the Secretary-General Pursuant to Para. 2 of Security Council Resolution 808 (I993), 3. Mai I993, S/25704, $\mathbb{S} 35,47$. 44 UN Sicherheitsratsresolution 955 (I994).

45 Zum Vergleich des IMT-Statuts mit dem Römischen Statut vgl. Andreas Zimmermann, Das juristische Erbe von Nürnberg - das Statut des Internationalen Mili-

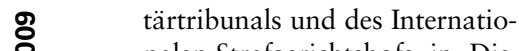
nalen Strafgerichtshofs, in: Die

wientribunals vom 28. Mai 1993 trägt deutlich sichtbar Züge des IMT-Statuts vom 8 . August I $945 .{ }^{43}$ Bereits ein Jahr später wurde ein weiteres Tribunal vom Sicherheitsrat der Vereinten Nationen zur strafrechtlichen Verfolgung des Völkermordes in Ruanda eingerichtet. ${ }^{44}$ Im Jahre 1998 schließlich verabschiedete eine internationale Konferenz in Rom das Statut für den Internationalen Strafgerichtshof (IStGH). Bereits vier Jahre später, am I. Juli 2002, konnte das Statut in Kraft treten und der IStGH seine Arbeit aufnehmen. ${ }^{45}$ Die Überzeugung, dass ein Kernbestand der Menschenrechte auch für jeden Staatsmann verbindlich ist und dass der Grundsatz der Staatssouveränität hinter den Schutz dieser Menschenrechte auch durch Strafrecht zurückzustehen hat, darf im Grundsatz weltweit als anerkannt gelten. ${ }^{46}$

\section{c) Internationale Strafzwecke von Nürnberg bis heute}

Es lässt sich also festhalten, dass der Nürnberger Prozess zum Ziel hatte, die internationale Verantwortlichkeit von Individuen für makrokriminelle Straftaten zu etablieren. Darüber hinaus verfolgte der Nürnberger Prozess das Ziel, den Automatismus von Krieg und Rache zu durchbrechen. Aber mehr noch als zur Verhinderung von Rache soll der internationale Strafprozess dazu beitragen, zukünftige Kriege insgesamt zu verhindern (»prevent future wars«). Die Präambel des Internationalen Strafgerichtshofs drückt dies in anderen Worten so aus: $»(. .$.$) entschlossen, der Straflosigkeit der$ Täter ein Ende zu setzen und so zur Verhütung solcher Verbrechen beizutragen «. Der den Amerikanern in Nürnberg besonders wichtige Bezug zur Strafbarkeit des Angriffskriegs erscheint in der modernen Fassung weiter. Der Abschreckungseffekt soll auf sämtliche internationalen Verbrechen Wirkung entfalten. Dem Grunde nach hat sich in der Ausrichtung und dem Anspruch internationaler Prozesse wenig geändert. In Europa fand seit dem Zweiten Weltkrieg keine weitere militärische Auseinandersetzung statt. Racheakte unter den ehemaligen Kriegsparteien sind nach den Verurteilungen von Nürnberg ausgeblieben. Allerdings müssen, bevor voreilig Kausalitäten behauptet und daraus Schlüsse für heutige Konfliktfelder gezogen werden, die Funktionsbedingungen in Nürnberg genau analysiert werden.

Nürnberger Prozesse. Völkerstrafrecht seit I945 (Fn. 2) 247-258.

46 Jedenfalls vor einem internationalen Straftribunal hat der Grundsatz der Immunität für Straftaten, die Staatenführer in Ausführung ihres öffentlichen Amtes begehen, keine prozesshindernde Wirkung mehr. Vor nationalen Spruchkörpern mag das anders sein, vgl. IGH D. R. Kongo vs. Belgien, Urteil vom I4. Februar 2002,
Reports 2002, I2 I; s. auch Claus KRESs, Der Internationale Gerichtshof im Spannungsfeld zwischen Völkerstrafrecht und Immunitätenschutz, in: GA 2003, 2543. Dazu im historischen Zusammenhang: GeOFFrey RoberTson, Ending Impunity: How International Criminal Law Can Put Tyrants on Trial, in: Cornell International Law Journal 38 (2005) 649-67I. 
(I) Rahmenbedingungen in Nürnberg

Im historischen Kontext sind im Bezug auf Nürnberg eine Reihe von Besonderheiten auffällig: (I) Deutschland war nach der bedingungslosen Kapitulation unter alliierter Kontrolle. Damit waren nicht nur die potentiellen Angeklagten in den Händen der Siegermächte, sondern es bot sich auch ungehinderter Zugang zu allen möglichen Beweismitteln. (2) Aufgrund dessen, aber auch aufgrund der besonderen Akribie bei der Aktenführung seitens der deutschen Verwaltung, stellte sich die Beweislage als besonders gut, geradezu erdrückend dar. (3) In der deutschen Gesellschaft war Strafrecht seit langem als sozialpsychologische Realität anerkannt. Strafrecht als soziales Reaktionsmodell für Verbrechen war für die Bevölkerung ebenso wie für die Angeklagten grundsätzlich akzeptiert. Auch wenn die Angeklagten, allen voran Hermann Göring, das Internationale Militärtribunal als Tribunal der Sieger ablehnten, beugten sie sich doch der Autorität des Gerichts und des vorsitzenden Richters Lord Lawrence. ${ }^{47}$ Die "Spielregeln « eines Strafverfahrens wurden deshalb insgesamt grundsätzlich eingehalten. (4) Der in der unmittelbaren Folge des Zweiten Weltkriegs entstandene Kalte Krieg zwang in der Folge der strafrechtlichen Aufarbeitung des NS-Regimes die Staaten Europas zu einem Prozess rascher Integration.

(2) Rahmenbedingung in späteren Konflikten

So günstige Rahmenbedingungen finden sich in anderen Konflikten, in denen strafrechtliche Aufarbeitung versucht wird, nicht. Das zeigte sich bereits bei der Errichtung des Jugoslawientribunals: Als 1993 das Tribunal seine Arbeit aufnehmen sollte, war der Bürgerkrieg noch in vollem Gange; er hatte noch nicht einmal seinen Höhepunkt erreicht. ${ }^{48}$ Eine abschreckende Wirkung ging von dem Tribunal daher offenbar nicht aus. Auch nach Beendigung des Ursprungskonflikts wurde die Strafverfolgung seitens der betroffenen Staaten nur sehr schleppend aufgenommen, und die Kooperation der internationalen Truppen beim Verhaften gesuchter Kriegsverbrecher ließ zu wünschen übrig. ${ }^{49}$ Bis heute ist der bosnisch-serbische General Mladić auf freiem Fuß. Dazu kam es im Jahr 1998 zu einer Verlagerung des Konflikts auf das Kosovo, was eine massive militärische Intervention seitens der Nato zur Folge hatte. ${ }^{5}$ Von einer Versöhnungsfunktion des Strafrechts kann in diesem Konflikt bis heute nicht gesprochen werden.

47 Vgl. Sitzungsprotokoll: Internationaler Militärgerichtshof, Amtlicher Text, Nürnberg I947, Band 2, II3.
48 Die Belagerung Sarajewos sowie die Massaker in Srebrenica folgten erst in den Jahren I993/94; zur Chronik des Krieges s. Matthias VetTer, Chronik der Ereignisse I986-2002, in: Der Jugoslawienkonflikt, hg. von Dunja Melčić, 2. Aufl., Wiesbaden 2007, 550577.

49 Vgl. die Zusammenstellung bei Christoph Safferling, Dunja MelČIĆ, Das Kriegsverbrecher- tribunal in Den Haag - Rechtsgrundlagen und Entwicklung, in: Der Jugoslawienkonflikt, hg. von Dunja Melčić, 2. Aufl., Wiesbaden 2007, 503-516.

$50 \mathrm{Zu}$ der sehr umstrittenen Frage der völkerrechtlichen Zulässigkeit dieser Intervention: BRUNO SIMMA, Nato, the UN, and the Use of Force: Legal Aspects, in: European Journal of International Law Io (I999) I. 
Ruanda, die zweite Situation, in der ein UN-Tribunal eingesetzt wurde, bietet wieder andere soziale und juristische Voraussetzungen. Hier war der zu verfolgende Konflikt zwar bei Errichtung des Tribunals wenigstens vordergründig abgeschlossen, die ruandische(n) Gesellschaft(en) bauen aber auf kommunale Reaktionsformen der Gacaca. ${ }^{5 \mathrm{I}}$ Darunter wird eine Art Gerichtsverfahren verstanden, das Schadensausgleich und andere Sanktionsformen miteinander verbindet und nicht in der dem westlichen Strafsystem bekannten Form zwischen Täter und Opfer unterscheidet.

Die am Internationalen Strafgerichtshof anhängigen Fälle sind ebenfalls kritisch zu beurteilen. Im Kongo herrscht weiter große Instabilität, in Uganda werden die internationalen Haftbefehle als abträglich für den Friedensprozess angesehen, in Darfur schließlich ist nicht nur der Konflikt außer Kontrolle, es ist auch eine Ermittlung vor Ort mangels Kooperation des Sudans unmöglich.

Ohne historisches Vorbild sind bislang die sog. hybriden Tribunale, die teils mit nationalem, teils mit internationalem Personal besetzt sind. ${ }^{52} \mathrm{Zu}$ erwähnen ist hier insbesondere das Sondergericht für Sierra Leone, das mit dem Verfahren gegen Charles Taylor seine schwierigste Aufgabe noch vor sich zu haben scheint. Kurioserweise zeigt sich hier ein als besonderer Vorteil gepriesenes Charakteristikum, dass nämlich bei hybriden Tribunalen die Strafverfolgung vor den Augen der betroffenen Bevölkerung im Land selbst stattfindet, als nicht durchführbar: Das Verfahren wird in den Räumen des Internationalen Strafgerichtshofs in Den Haag abgehalten. ${ }^{53}$ Noch ausgeprägter ist die nationale Komponente in den Sonderkammern in Kambodscha (Extraordinary Chambers in the Courts of Cambodia). ${ }^{54}$ In der Folge ist der Verdacht der politischen Einflussnahme seitens der kambodschanischen Regierung kaum auszuräumen. Weit verbreitete Korruptionsvorwürfe haben das Vertrauen in die Gerichtsbarkeit allgemein unter der Bevölkerung seit langem beschädigt. Ein durchschlagender Erfolg ist die Hybridstruktur nicht und dürfte sich angesichts der Konsolidierung des Internationalen Strafgerichtshofs eher wieder auf dem Rückzug befinden. ${ }^{55}$

Dieser kursorische Überblick soll zeigen, dass die Funktionsbedingungen in den einzelnen Anwendungsgebieten von Völkerstrafrecht seit Nürnberg erheblich variieren. Die einfache Schlussfolgerung, dass das, was hier funktioniert hat, auch dort funktionieren muss, ist naiv. Eher im Gegenteil: Die soziale Akzeptanz

5 I Vgl. Mark Drumbl, Atrocity, Punishment, and International Law, Cambridge 2007, 85-98.

52 Vgl. Daphna Shraga, The Second Generation UN-Based Tribunals: A Diversity of Mixed Jurisdiction, in: Internationalized Criminal Courts, hg. von Cesare Romano, André Nollkaempfer und JanN KLEFFNER, Oxford 2004, I 5-38.

53 Vgl. David Cohen, Hybrid Tribunals: Prospects and Challenges, in: Historische Dimensionen von Kriegsverbrecherprozessen nach dem Zweiten Weltkrieg (Fn. I 2) 9I-I07.

54 Überblick bei: MichaEl BoHLANDER und RENATE WINTER, Internationalisierte Strafgerichte auf nationaler Ebene - Kosovo, Kambodscha, Sierra Leone und Timor-Leste, in: Internationale Strafgerichtshöfe, hg. von STEFAN
KIRSCH, Baden-Baden 2005, 26I279, $267 \mathrm{ff}$.

55 Eine positive Bestandsaufnahme der hybriden Struktur findet sich bei Frédéric MÉgret, In Defense of Hybridity: Towards a Representational Theory of International Criminal Justice, in: Cornell International Law Journal 38 (2005) 725-75I. 
von Strafrecht als angemessene und abschließende Reaktion auf massive Menschenrechtsverletzungen muss ebenso gewährleistet sein, wie die praktische Durchführbarkeit von Strafverfahren sichergestellt werden muss. Ohne flankierende Versöhnungsmaßnahmen wird sich außerdem ein Erfolg in Form einer nachhaltigen Friedfertigkeit unter vormaligen Konfliktparteien nicht einstellen können. Von Nürnberg lernen heißt demnach auch, den Blick auf die strukturellen Bedingungen zu wagen und die rechtlichen Reaktionsmuster entsprechend anzupassen.

2. Die Internationalen Kernverbrechen

a) Die »Nürnberger Verbrechen «

Die Signifikanz des Nürnberger Hauptkriegsverbrecherprozesses als des ersten internationalen Strafprozesses besteht außerdem darin, dass im IMT-Statut eine Liste internationaler Kernverbrechen aufgestellt worden ist, die bis heute dem Grunde nach Gültigkeit hat. Dazu zählen: (I) das Verbot des Angriffskrieges bzw. Verbrechen gegen den Frieden, wie es in Art. 6 a IMT-Statut heißt, (2) Kriegsverbrechen, Art. 6 b IMT-Statut, und (3) Verbrechen gegen die Menschlichkeit, normiert in Art. 6 c IMT-Statut. Diese Liste internationaler Verbrechen stellt in der Menschheitsgeschichte ein absolutes Novum dar. Entsprechend war die Anerkennung dieser Verbrechenstatbestände im Verlaufe des Nürnberger Prozesses wie auch in der Nachwirkung höchst umstritten. Das gilt vor allem für den zuerst genannten Tatbestand des Verbrechens gegen den Frieden. Zwar gab es im sog. Briand-Kellogg-Pakt von 1928 eine vertragliche Grundlage für die Ächtung des Krieges in der internationalen Politik; ein strafrechtliches Verbot war damit allerdings nicht verbunden. Im Vergleich dazu war der zweite Tatbestand, die Kriegsverbrechen, relativ unstreitig. Auf der Grundlage verschiedener Genfer Konventionen ebenso wie der Haager Landkriegsordnung von 1907 gab es gewisse Handlungen, die als Mittel und Methode der Kriegsführung bei Androhung von Strafe verboten waren. Eine völlige Neuschöpfung hingegen bilden die Verbrechen gegen die Menschlichkeit. Dieser Verbrechenstatbestand stellt den Versuch dar, die bis zum damaligen Zeitpunkt ungekannte Grausamkeit und Systematik in der Vernichtung von Menschenleben in einen justiziablen Straftatbestand zu gießen. ${ }^{56}$ Die sich

\footnotetext{
56 Report of the Secretary-General Pursuant to Para. 2 of Security Council Resolution 808 (I993), 3. Mai I993, S/25704, $\mathbb{4} 4$.
} 
dahinter verbergenden Straftaten, wie Mord, Folter, Versklavung, Ausrottung, sind in sämtlichen Staaten der Welt als strafbare Handlungen zweifellos anerkannt. Fraglich ist indes, wie man zum Ausdruck bringt, dass diese »einfachen Verbrechen « in einem Kontext geschehen sind, der einen Angriff auf die Menschheit als Ganzes enthält. Im Nürnberger Verfahren war man in dieser Kategorie relativ zurückhaltend, und der Tatbestand fand nur im Zusammenhang mit einer kriegerischen Handlung Anwendung. Lediglich zwei Angeklagte wurden vom IMT ausschließlich wegen Verbrechen gegen die Menschlichkeit verurteilt: Streicher erhielt die Todesstrafe, von Schirach wurde mit 20 Jahren Haft bestraft.

Das bedauerliche Resultat dieser restriktiven Handhabung lag darin, dass Verbrechen an der deutschen Bevölkerung, insbesondere so wie sie etwa in der Reichspogromnacht verübt wurden, im Nürnberger Hauptkriegsverbrecherprozess nicht thematisiert wurden. ${ }^{57}$ Gleichwohl war im Nürnberger Statut mit der Formulierung der Verbrechen gegen die Menschlichkeit die begriffliche, aber auch die juristische Grundlage für diesen neuen zusammenfassenden internationalen Tatbestand gelegt. Im Kontrollratsgesetz Nr. Io wurde der Tatbestand geweitet und der Begehungszusammenhang mit dem Krieg als Voraussetzung gestrichen. ${ }^{58}$

\section{b) Der Sonderfall »Völkermord «}

In dieser Liste der internationalen Verbrechen fällt auf, dass der Völkermord nicht enthalten ist. Der Tatbestand des Völkermordes war im Jahre I945 juristisch nicht existent. Erst drei Jahre später, am 9. Dezember 1948, gelang es, durch eine Resolution der Generalversammlung der Vereinten Nationen die Konvention zur Verhütung und Bestrafung von Völkermord zu verabschieden. ${ }^{59}$ Dieser Straftatbestand knüpft an die Verbrechen gegen die Menschlichkeit an, betont dabei im Speziellen die Vernichtung einer besonders, und zwar national, ethnisch, rassisch oder religiös, abgrenzbaren Gruppe. Die Konvention und damit den Tatbestand des Völkermordes verdankt die Welt dem unermüdlichen Engagement des polnischen Juristen Raphael Lemkin (I900I959). ${ }^{60}$ Raphael Lemkin selbst war im Jahr I939 die Flucht über Skandinavien in die USA gelungen, er verlor aber fast seine ganze Familie im Holocaust. ${ }^{6 \mathbf{I}}$ Nach Kriegsende diente er zeitweise im US-amerikanischen Anklägerteam des obersten Bundesrichters

57 In der sehr auf die »Verschwörung « abhebenden Eröffnungsrede von Robert H. JACKSON fand die Reichspogromnacht zwar Erwähnung, nicht aber im Urteil; vgl. Internationaler Militärgerichtshof, Amtlicher Text, Nürnberg 1947, Band 2, I 20.

58 Official Gazette of the Control Council for Germany, No. 3 , p. 22; vgl. dazu KaI Амвos, Der Allgemeine Teil des Völkerstraf- rechts, Berlin 2002, 83; zuletzt auch ausführlich STEFAN KIRSCH, Zweierlei Unrecht - Zum Begehungszusammenhang der Verbrechen gegen die Menschlichkeit, in: Festschrift für Rainer Hamm, hg. von Regina Michalke, Wolfgang Körberer, Jürgen Pauly und Stefan KIRSCH, Berlin 2008, 269-288.

59 Vgl. BGBl. II I954, 730; dazu und $\mathrm{zu}$ Vorentwürfen insbesondere
William Schabas, Genozid im Völkerstrafrecht, Hamburg 2003, 7 IO ff.

60 Vgl. Raphael Lemkin, Axis Rule in occupied Europe. Laws of Occupation, Analysis of Government, Proposals for Redress, Washington I944; DERs., Genocide as a Crime under International Law, in: American Journal of International Law 4I (I947) I45-I 5I.

6I Zur wenig erschlossenen Biographie s. John CoOper, Raphael Lemkin and the Struggle for the Genocide Convention, New York 2008. 
62 In der Anklageschrift ist Völkermord auf Drängen Lemkins definiert als: »the extermination of racial and national groups, against the civilian populations of certain

Robert H. Jackson und versuchte dort, seine Idee eines Völkermordtatbestandes $\mathrm{zu}$ verwirklichen, ohne sich aber mit dieser neuartigen Herangehensweise durchsetzen zu können. ${ }^{62}$ Erst drei Jahre später gelang ihm der Durchbruch. Heute steht der Völkermord in Art. 6 des Römischen Statuts für den internationalen Strafgerichtshof an erster Stelle der internationalen Kernverbrechen. So hat der Völkermord historisch zwar seinen Ursprung in den Verbrechen der Nationalsozialisten an der jüdischen und an anderen Minderheiten, er ist juristisch in der strafrechtlichen Aufarbeitung in der unmittelbaren Kriegsfolge allerdings nicht zur Anwendung gelangt.

Damit wird auch ein weiterer Umstand deutlich: Im Nürnberger Hauptkriegsverbrecherprozess ging es vornehmlich - der US-amerikanischen Anklagedoktrin entsprechend - um die Ahndung des Führens eines Angriffskriegs. Der Holocaust, die Vernichtung von Minderheiten und »menschenunwürdigen « Lebens, trat demgegenüber eher in den Hintergrund. Das entspricht nicht unserer heutigen Wahrnehmung, erklärt sich aber aus den soeben skizzierten juristischen Rahmenbedingungen und dem Umstand, dass das wahre Ausmaß der Vernichtungsmaschinerie in Auschwitz und anderswo erst im Laufe des Prozesses bzw. erst einige Jahre später mit der Auffindung des sog. Wannsee-Protokolls im Jahre I947 deutlich wurde. ${ }^{63}$

\section{c) Moderne Perspektiven}

Es mag als Ironie der Geschichte angesehen werden, dass vor allem die US-amerikanische Regierung im letzten Jahrzehnt die Verabschiedung eines Straftatbestandes der Aggression auf internationaler Ebene erfolgreich verhindert hat. In Nürnberg war es doch gerade dieser Tatbestand, den das US-amerikanische Anklägerteam um Robert Jackson für alle Zukunft festgestellt haben wollte. ${ }^{64}$ Für das nach Art. 5 Abs. I (d), 2 IStGHSt vorgesehene Verbrechen der Aggression konnte noch keine konsensfähige Definition gefunden werden. ${ }^{65}$ Die moderne Tatbestandsfassung der Verbrechen gegen die Menschlichkeit, etwa in Art. 7 IStGHSt, sieht den internationalen Charakter des Delikts dadurch erfüllt, dass die Einzeltat im Zusammenhang mit einem ausgedehnten oder systematischen Angriff auf eine Zivilbevölkerung stehen muss. ${ }^{66}$ Ein Krieg bzw. ein bewaffneter Konflikt ist hingegen nicht (mehr)

occupied territories in order to destroy particular races and classes of people, and national, racial, or religious groups, particularly Jews, Poles, and Gypsies and others «, Internationaler Militärgerichtshof, Band 2, 45, 59. Rechtsdogmatisch erscheint dies im Zusammenhang mit der Misshandlung der Bevölkerung in besetzen Gebieten, also als Kriegsverbrechen.
63 Es wurde im sog. Wilhelmstraßenprozess aktenkundig, vgl. KASTNER (Fn. I I) 268.

64 Internationaler Militärgerichtshof, Amtliche Sammlung, Nürnberg I947, Band 2, I 53.

65 Zusammenfassend mit weiteren

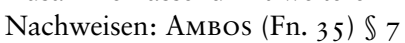
Rn. 25 I-258a.

$66 \mathrm{Zu}$ den einzelnen Voraussetzungen s. Амвоs (Fn. 35) $\mathbb{7}$ Rn. I82I98. 
erforderlich. Die größte Veränderung ist bei den Kriegsverbrechen zu beobachten. Seit der ersten Entscheidung der Berufungskammer des Jugoslawientribunals im Fall gegen Duško Tadić $^{67}$ finden diese in großem Umfang auch im nicht internationalen bewaffneten Konflikt Anwendung. ${ }^{68}$

\section{Der »Allgemeine Teil« des Völkerstrafrechts}

Neben der Formung einer verbindlichen Liste an Verbrechenstatbeständen lassen sich auch gewisse Maßstäbe für den Allgemeinen Teil des Strafrechts dem Nürnberger Verfahren entnehmen, die bis heute nachwirken. Anhand von vier Zurechnungsfeldern (Vorsatz, Mittäterschaft, Vorgesetzten-Untergebenenverhältnis und Strafzumessung) soll diese These exemplarisch untermauert werden.

\section{a) Nürnberger Zurechnungsformen}

Dass nur vorsätzliches Verhalten bestraft werden kann, hat Justice Jackson in seiner Eröffnungsrede bereits klargestellt. »We charge guilt on planned and intended conduct that involves moral as well as legal wrong. " ${ }^{69}$ Dies galt als Selbstverständlichkeit und bedurfte daher keiner expliziten Erwähnung. ${ }^{70}$ In den Einzelheiten war im Nürnberger Verfahren allerdings wenig zu erinnern. Die Beweisführung konnte sich im Wesentlichen auf vorhandene Dokumente stützen, von deren Inhalt die Angeklagten schon deshalb Kenntnis hatten, weil diese ihre Unterschrift trugen. ${ }^{7 \mathbf{I}}$

Das Statut des IMT enthielt in Art. 6 (a) eine besondere Form der Mittäterschaft, die sog. Conspiracy, Verschwörung zum Angriffskrieg. Auf andere Verbrechen, etwa Kriegsverbrechen, wurde das Konzept der Verschwörung entgegen den Vorstellungen des Chefanklägers Jackson vom IMT nicht angewendet. ${ }^{72}$ Über das Verschwörungsmodell lässt sich auf recht schlichte Art eine Zurechnung konstruieren, da es auf das Ausführungsstadium der Tat nicht ankommt, weil der gemeinsame Plan für die täterschaftliche Zurechnung ausreicht. ${ }^{73}$ Mit dem herkömmlichen Verständnis des Schuldprinzips ist dies kaum mehr zu vereinbaren ${ }^{74}$ allerdings verringern sich beweistechnische Schwierigkeiten bezogen auf den jeweiligen Tatbeitrag der Mittäter. So wurden beispielsweise in Nürnberg auch die obersten Militärs wie Raeder, Keitel und Jodl

67 ICTY Prosecutor v. Duško Tadić, Berufungskammer, 2.10.1995, $\int \mathbb{S} 71-137$.

68 Diese Entscheidung wird immer noch sehr kritisch gesehen, vgl. Alexander ZaHAR und Goran SLuiter, International Criminal Law, Oxford 2008, I I 2, I 54, und findet sich in Art. 8 Römisches Statut auch nur in eingeschränktem Umfang wieder.
69 JACKSON im Eröffnungsplädoyer, Internationaler Militärgerichtshof, Amtliche Sammlung, Nürnberg I947, Band 2, I 53.

70 Albin Eser, Mental Elements Mistake of Fact and Mistake of Law, in: The Rome Statute of the International Criminal Court: A Commentary, hg. von ANTONIO Cassese, Paola Gaeta und John JoNES, Oxford 2002, 889, $893 \mathrm{ff}$.
7I Der Nürnberger Prozess wurde gelegentlich - etwa von REBECCA West, A Train of Powder, Chicago 2000, 3 - auch als »Hochburg der Langeweile « bezeichnet.

72 Robert Herde, Command Responsibility, Baden-Baden 200I, I72.

73 Robert Cryer, Prosecuting International Crimes, Cambridge 2005, 309-3II.

74 Vgl. Christoph Safferling, Vorsatz und Schuld, Tübingen 2008, $408 \mathrm{f}$. 
$75 \mathrm{Zu}$ diesem Zusammenhang auch: Otto Triffterer, in: Commentary on the Rome Statute of the International Criminal Court, hg. von Otto Triffterer, 2. Aufl., München 2008, Art. 33 Rn. 32; s. auch Frank Neubacher, Kriminologische Grundlagen einer Internationalen Strafgerichtsbarkeit, Tübingen 2005, 430-434. 76 Allgemein $\mathrm{zu} »$ Command Responsibility « in Nachkriegsprozessen gegen deutsche und japanische Generäle, vgl. Herde (Fn. 72).

77 Im Tokioter Prozess mag das anders gewesen sein, vgl. CRYER (Fn. 73), 3I6f. Als »leading case « in diesem Punkt gilt gemeinhin das Verfahren gegen General Yamashita vor einem US Military Tribunal in Manila, s. UNWCC, Law Reports of Trials of War Criminals, Vol. IV, I ff. Zum »Röchling «-Fall eines französischen Besatzungsgerichts in Rastatt vgl. Henning Radtke, Gedanken zur Vorgesetztenverantwortlichkeit im nationalen und internationalen Strafrecht, in: Festschrift für Egon Müller, hg. von HeIKe JUNG, Bernd Luxenburger und EberHARD WAHLEN, Baden-Baden 2008, 577-592.

78 Etwa im Nacht- und Nebelerlass. Zur Zurechnung in diesem Punkt vgl. HARris (Fn. I6) 2I3-2I7.

einfach deshalb der Verschwörung zum Angriffskrieg für schuldig angesehen, weil ihnen die NS-Politik der Schaffung neuen Lebensraums im Osten bekannt war. Unter den Militärs gelang es einzig Dönitz, sich dieser argumentativen Schlinge zu entziehen.

Im Rahmen der Schuld ist es vor allem das Verhältnis zwischen Vorgesetztem und Untergebenem, das in zweifacher Hinsicht zu strafrechtlichen Zurechnungsproblemen führt. ${ }^{75}$ Einerseits ist unklar, ob der Vorgesetzte für die Taten seiner Untergebenen verantwortlich ist, von denen er wusste und die er hätte verhindern können (Vorgesetztenverantwortlichkeit oder »Command Responsibility «). ${ }^{76}$ Im Nürnberger Verfahren ist dieses Modell kaum thematisiert worden. ${ }^{77}$ Das liegt vor allem daran, dass sich in der Beweisführung eine Befehlskette zu den Angeklagten herstellen lie $\beta^{78}$ und das Zurechnungskonzept der Vorgesetztenverantwortlichkeit als Auffangkonstrukt sich damit erübrigte. ${ }^{79}$ Andererseits stellt sich die Frage, ob sich der Untergebene auf einen Befehl berufen kann, um der strafrechtlichen Verfolgung zu entkommen (Handeln auf Befehl oder »Superior Order «). Hier wiederum bot die hierarchische Struktur des NS-Regimes mit dem »Führer« als alleinigem Befehlshaber einen idealen Ansatzpunkt, die im Nürnberger Verfahren Angeklagten zu exkulpieren. ${ }^{8 \circ}$ Dieser Entschuldigungsargumentation stand aber Art. 8 IMT-Statut im Weg. Danach konnte die Berufung auf einen Befehl allenfalls strafmildernde Wirkung haben und niemals zum Ausschluss der Strafbarkeit führen. Auch eine Strafmilderung wurde im Urteil von Nürnberg abgelehnt, da »derart empörend und weitverbreitete Verbrechen bewusst, rücksichtslos und ohne militärische Notwendigkeit oder Rechtfertigung begangen worden sind $\ll{ }^{8 \mathrm{x}}$ In diesen Fällen hat der Angeklagte immer die Wahl (»moral choice «) sich gegen das Unrecht und für das Recht zu entscheiden. ${ }^{82}$

Die Frage der Strafzumessung erwies sich auch in Nürnberg als delikat. Das IMT scheute sich nicht, in Ermangelung konkreter Strafrahmen ${ }^{83}$ gegen die Angeklagten entsprechend ihrer jeweiligen Schuld unterschiedliche Sanktionen zu verhängen (Art. 27 IMT-Statut): ${ }^{84}$ Während Göring in allen Anklagepunkten für schuldig angesehen und zum Tode durch den Strang verurteilt wurde, erhielt $\mathrm{He} ß$ lediglich eine lebenslange Freiheitsstrafe für Verschwörung zum Angriffskrieg. Julius Streicher wurde allein wegen Verbrechen gegen die Menschlichkeit zum Tode verurteilt. Sympathie und Antipathie als unausgesprochenes Strafzumes-

79 Als »default liability « bezeichnet von KaI Ambos, in: Commentary on the Rome Statute of the International Criminal Court, hg. von Otto Triffterer, 2. Aufl., München 2008, Art. 25 Rn. 49.

80 Vgl. Амвоs, Der Allgemeine Teil (Fn. 58) I I7; Gerhard Werle, Völkerstrafrecht, 2. Aufl., Tübingen 2007, Rn. 54I.
8I Internationaler Militärgerichtshof, Amtliche Sammlung, Nürnberg I947, Urteil, Band I 29I.

82 Ibid. 224.

83 Heiko Ahlbrecht, Geschichte der völkerrechtlichen Strafgerichtsbarkeit im 20. Jahrhundert, Baden-Baden I999, 92.

84 Gisela Manske, Verbrechen gegen die Menschlichkeit als Verbrechen gegen die Menschheit, Berlin 2003, 67. 
sungskriterium werden hier, aber auch bei Albert Speer deutlich, der mit einer zwanzigjährigen Haftstrafe vergleichsweise milde behandelt wurde. ${ }^{85} \mathrm{Zu}$ konzedieren ist immerhin, dass bei der Abstimmung unter den Richtern eine große Einmütigkeit zu verzeichnen war. $^{86}$

\section{b) Zurechnungskonzepte moderner Gerichte}

Im modernen internationalen Strafprozess ist das Erfordernis einer subjektiven Zurechenbarkeit des Verhaltens allgemein anerkannt. In Art. 30 IStGHSt werden die Erfordernisse an die subjektive Tatseite zusammengefasst, ohne dass dadurch unterschiedliche Herangehensweisen zwischen dem mens rea-Konzept des angloamerikanischen Rechtskreises und etwa dem deutschen Vorsatzkonzept der herrschenden Schuldtheorie harmonisiert werden. ${ }^{87}$ Die zunehmende Komplexität der Normen sowie die hohen Beweisanforderungen führen zu einer Tendenz in der Rechtsprechung internationaler Tribunale, das kognitive Element in den Mittelpunkt zu stellen. ${ }^{88}$ Derartige Ansätze fanden sich wegen der besonderen Beweissituation bereits im Nürnberger Verfahren.

Die moderne völkerstrafrechtliche Zurechnung verzichtet auf das Konzept der »conspiracy «. Allerdings hat die internationale Rechtsprechung am Jugoslawientribunal ein Zurechnungsmodell entwickelt, das stark an die "Verbrechensverschwörung " erinnert. ${ }^{89}$ Dieses Modell firmiert unter dem Namen »joint criminal enterprise « (jce) und erscheint in drei Formen. ${ }^{90}$ Während in den ersten zwei Erscheinungsformen klar ein mittäterschaftliches $\mathrm{Zu}$ rechnungsmodell auf der Grundlage eines gemeinsamen Tatplans und einer arbeitsteiligen Tatausführung erkennbar ist, sieht die jce III jeden als Mittäter, der an einem gemeinsamen Tatplan teilhat, unabhängig davon, ob die einzelnen Taten noch von diesem Plan gedeckt sind. Was nach $\mathbb{} 25$ Abs. 2 StGB als klarer Mittäterexzess anzusehen wäre, wird hiernach zugerechnet mit dem pragmatischen Argument, dass bei systematischen Verbrechen gleichsam von vorneherein mit überschießenden Taten gerechnet werden müsse. Der gemeinsame Plan (»common purpose «) tritt demnach so stark in den Vordergrund, dass der Vergleich mit der Verbrechensverschwörung als eigentlichem Strafgrund auf der Hand liegt. Inwieweit der Internationale Strafgerichtshof diese Zurechnungsform vom Jugoslawientribunal übernimmt, ist bislang unklar. Erste
85 Das hat der US-Ankläger Whitney Harris in verschiedenen Gesprächen mir gegenüber offen eingeräumt.

86 Bradley Smith, Der Jahrhundertprozess, Frankfurt a. M. I977, I90.

87 Die Unterschiede kulminieren in der Differenzierung zwischen recklessness auf der einen und dolus eventualis auf der anderen Seite. Dazu ICTY Prosecutor v.
Tihomir Blaškić, Berufungskammer, Urteil vom 27.9.2005, $\mathbb{S} 27$, 34 ff. Ähnlich auch ICC Prosecutor v. Thomas Lubanga, Confirmation of Charges, 29.I.2007, $\int \mathbb{3}$ 52-355; grundsätzlich zur Unterscheidung SAFFERLING (Fn. 74) 360-368, 483 .

88 Vgl. Werle (Fn. 80) Rn. 355.

89 ICTY Prosecutor v. Tadić, Beru-

fungskammer, Urteil vom I 5.7. I999, $\mathbb{S} \mathbb{S}_{72} \mathrm{ff}$.; bestätigt in:
Prosecutor v. Krstić, Berufungskammer, Urteil vom I9.4.2004 (IT-98-33-A), \237 u. a. Vgl. die Nachweise bei Амвоs (Fn. 35) \ 7 Rn. 30 Fn. I I9.

90 Dazu Verena HaAn, Joint Criminal Enterprise, Berlin 2008, 248304, die allerdings das Conspiracy-Modell des IMT als historischen Anknüpfungspunkt nicht behandelt. 
9I IStGH Prosecutor v. Thomas Lubanga, Confirmation of Charges, 29.I.2007, $\mathbb{S} 3 \mathrm{I} 9 \mathrm{ff}$.; danach bedarf es für die Mittäterschaft nach Art. 25 (3)(a) IStGHSt eines Tatplans und einer gemeinsamen Tatbeherrschung.

92 Vgl. Kai Амвоs, Superior Responsibility, in: The Rome Statute of the International Criminal Court: A Commentary, hg. von antonio Cassese, Paola Gaeta und JOHN JONES, Oxford 2002, $824 \mathrm{ff}$.

93 Vgl. dazu Werle (Fn. 80) Rn. 487-49I.

94 OtTo Triffterer, in: Commentary on the Rome Statute of the International Criminal Court, hg. von Otto Triffterer, 2. Aufl., München 2008, Art. 33 Rn. 30. 95 Werle (Fn. 80) Rn. 552.

96 Soweit ersichtlich beschäftigt sich bislang nur eine einzige Monographie mit übergreifenden international verbindlichen Kriterien: Jan Nemitz, Strafzumessung im Völkerstrafrecht, Freiburg 2002.

\& 97 Vgl. etwa zusammenfassend: ICTY Prosecutor v. Kupreškić

Anzeichen deuten in eine eher gemäßigte Richtung und auf einen starken Einfluss deutscher Strafrechtswissenschaft hin. ${ }^{9 \mathbf{I}}$

Die Konzepte der Vorgesetztenverantwortung und des Handelns auf Befehl sind auch im modernen Völkerstrafrecht höchst relevant und dogmatisch umstritten. So ist eine »command responsibility « völkergewohnheitsrechtlich dem Grunde nach anerkannt; ${ }^{92}$ unklar ist allerdings, ob über dieses Zurechnungsmodell auch fahrlässige Aufsichtspflichtverletzungen seitens des Vorgesetzten zu einer Haftung für die vorsätzliche Tat des Untergebenen führen können, wie dies von Art. 28 IStGHSt vorgesehen ist. Mit dem Schuldprinzip wird das schwerlich zu vereinbaren sein, weshalb auch im VStGB ein anderer Weg gegangen und in $\mathbb{S} \mathbb{S} \mathrm{I}_{3}, \mathrm{I}_{4}$ VStGB eigene Tatbestände für die Strafbarkeit des Vorgesetzten geschaffen wurden. ${ }^{93}$

Hinsichtlich der Berufung auf einen Handlungsbefehl folgt Art. 33 IStGHSt nicht der strikten Lesart des IMT-Statuts. Der zur Befolgung des Befehls gesetzlich verpflichtete Täter ist dann entschuldigt, wenn er nicht wusste, dass die Anordnung rechtswidrig war, und die Rechtswidrigkeit des Befehls auch nicht offensichtlich war. In Absatz 2 wird freilich klargestellt, dass Anordnungen zu Völkermord oder zu Verbrechen gegen die Menschlichkeit stets offensichtlich rechtswidrig sind. Nur bei Kriegsverbrechen ist demnach Raum für eine strafbefreiende Wirkung eines Befehls. ${ }^{94}$ Eine Strafmilderung bei Handeln auf Befehl scheint über Art. 78 Abs. I IStGHSt möglich, ist aber nicht zwingend. ${ }^{95}$

Die Regelungen über die Strafzumessung können im Völkerstrafrecht nur als » unterkomplex « bezeichnet werden. ${ }^{96}$ Klarheit herrscht lediglich in Bezug auf die kategorische Ablehnung der Todesstrafe (Art. 77 IStGHSt). Zwar gibt es Andeutungen hinsichtlich einer eklektischen Orientierung an verschiedenen Strafzwecken wie Vergeltung, Abschreckung oder Spezialprävention, ${ }^{97}$ eine präzise Ausformung von Strafrahmen oder Strafzumessungskriterien steht aber noch aus. ${ }^{98}$ Deutlich wird dabei vor allem die Schwierigkeit, das »Unfassbare « in dem Zahlensystem der Freiheitsstrafe einzufangen. ${ }^{99}$

\section{Das Völkerstrafprozessrecht}

Eine große Herausforderung für das internationale Strafrecht bildet das Strafverfahrensrecht. ${ }^{\text {IoO }}$ Im Vorfeld des Nürnberger

et al., Verfahrenskammer, Urteil vom I4.I.2000, $\mathbb{} 848$ f.; vgl. zu den Strafzwecken etwa Frank Neubacher, Kriminologische Grundlagen einer internationalen Strafgerichtsbarkeit, Tübingen 2005, 422-430.

98 Zur Praxis der Ad-hoc-Tribunale zuletzt zusammenfassend: INÉs Weinberg de Roca und ChrisTOPHER M. RASSI, Sentencing and Incarceration in the Ad Hoc Tri- bunals, in: Stanford Journal of International Law 44 (2008) I62.

99 Vgl. Hanna Arendt in: Dies. und KARL JASPERS, Briefwechsel I926-I969, Zürich I993, 202.

Io० Einen Überblick über die einzelnen Schwierigkeiten findet sich bei Christoph Safferling, Towards an International Criminal Procedure, Oxford 200I. 
Hauptkriegsverbrecherprozesses war es unter den alliierten Siegermächten mehr als strittig, ob tatsächlich ein rechtsstaatlich ausgestattetes Strafverfahren angewendet werden sollte oder ob nicht eine summarische Prüfung der Schuld völlig ausreichend sei. Zudem wurde die Schuld der Angeklagten im Grunde als bewiesen und feststehend angesehen. Es ist vor allem das Verdienst des amerikanischen Chefanklägers Robert H. Jackson, dass in Nürnberg schließlich der Versuch unternommen wurde, ein Strafverfahren durchzuführen, das rechtsstaatlichen Anforderungen genügen kann. Entscheidet man sich für ein Strafverfahren, so Robert Jackson in seiner Rede vor der amerikanischen Gesellschaft für internationales Recht am I3. April I945, so muss grundsätzlich von der Unschuld der Angeklagten ausgegangen werden. Ist man nicht bereit, den Angeklagten freizusprechen, so darf man kein rechtsstaatliches Strafverfahren durchführen:

»That is one of the risks that are taken whenever trials are commenced. The ultimate principle is that you must put no man on trial under the forms of judicial proceedings if you are not willing to see him freed if not proven guilty. ${ }^{\text {Ior }}$

Im Nürnberger Hauptkriegsverbrecherprozess wurde dieser Grundsatz, bei aller Kritik im Einzelnen, ernst genommen, was man auch daran ablesen kann, dass mit von Papen, Fritsche und Neurath gegen den erbitterten Widerstand der sowjetischen Richter immerhin drei Personen freigesprochen worden sind. Robert Jackson war im Übrigen von der Mühe, die ein internationales Strafverfahren mit sich bringt, selbst überrascht, da er bei den Vorbereitungen des Prozesses im Sommer I945 noch davon ausging, dass bis Weihnachten die Verurteilungen vorlägen. Dass es schließlich noch weitere zehn Monate dauerte, ist aus heutiger Sicht gleichwohl überraschend, bedenkt man, dass etwa die Verfahren vor dem Jugoslawientribunal in der Regel mehrere Jahre beanspruchen. Auch wenn das Strafverfahren in Nürnberg nach modernen Maßstäben an Fairness einiges zu wünschen übrig ließ, muss doch berücksichtigt werden, dass den Angeklagten jeweils ein Verteidiger zur Seite gestellt wurde, dass ihnen ausreichend Zeit und Gelegenheit gegeben wurde, Beweise, die zur Entlastung dienen, vorzutragen und ein aufwendiges Dolmetschersystem die Verständlichkeit gewährleistete. Die Internationale Gemeinschaft sitzt im Bezug auf die Einhaltung der Fairnessstandards im Glas-

ior Robert H. Jackson, I3. April I945, Washington D. C. American Society of International Law, in: American Bar Association Journal 3I (I945) 290-294, 293. 
haus, und auch am Internationalen Strafgerichtshof muss der Ankläger immer wieder an die Angeklagtenrechte erinnert werden. ${ }^{102}$

\section{Zusammenfassung}

Die Signifikanz von Nürnberg zeigt sich vor allem in vier Punkten: Es gibt eine Liste von Kernverbrechen - Völkermord, Verbrechen gegen die Menschlichkeit, Kriegsverbrechen und Verbrechen des Angriffskriegs -, die gegenüber jeder Person Gültigkeit hat und die nach allgemeinen Zurechnungskriterien und mittels eines fairen Verfahrens vor internationalen Richtern durchgesetzt werden kann. Der Nürnberger Hauptkriegsverbrecherprozess hat in der unmittelbaren Nachkriegszeit nicht nur weltweit über I0.000 nationale Strafprozesse ausgelöst, sondern hat auch ein Bemühen in Gang gesetzt, Menschlichkeitsverbrechen unabhängig von Raum und Zeit strafrechtlich zu ahnden. Diese Verfahren sind in Europa Teil der kollektiven Erinnerungskultur. Dazu gehört die Erinnerung an die Verbrechen selbst, zugleich aber auch an die Möglichkeit, mit staatsgeleiteter Kriminalität angemessen umzugehen und in rechtsstaatlichen Strafverfahren eine Antwort zu finden. Ausgehend von Nürnberg hat sich daher die Meinung durchgesetzt, dass Völkerstrafrecht der Beginn eines Neuanfangs sein kann, der es überflüssig macht, auf Reaktionsmuster wie Rache und Krieg zurückzugreifen. Dabei darf man nicht übersehen, dass in Nürnberg vergleichsweise ideale Verhältnisse herrschten. Der Krieg war zu Ende, das rechtskulturelle Umfeld war gegenüber einem strafgerichtlichen Verfahren aufgeschlossen, der Großteil der Bevölkerung sah in den Angeklagten die Verantwortlichen für Krieg und Leid und befürwortete daher eine Verurteilung. Diese Grundbedingungen sind allerdings eher selten. In Jugoslawien hatte der Bürgerkrieg noch nicht einmal seinen Höhepunkt erreicht, als der Sicherheitsrat das Jugoslawientribunal einrichtete. In Ruanda besteht ein ausgeprägtes alternatives Reaktionsmodell, was mit strafrechtlicher Aufarbeitung kaum vergleichbar ist, in Kambodscha gibt es wenig Vertrauen in Gerichtsverfahren. Die Nürnberger Prozesse zeigen zwar einen - und in dem konkreten Fall, wie ich finde, erfolgreichen - Weg, grausames Unrecht aufzuarbeiten und ein friedliches Zusammenleben in der Zukunft zu gewährleisten, er ist aber keinesfalls der einzige und

IO2 Das erste Hauptverfahren vor dem

ICC musste vorläufig eingestellt werden, weil der Ankläger dem Angeklagten das Beweismaterial nicht in ausreichendem Umfang zur Verfügung gestellt hatte; vgl. ICC Prosecutor v. Lubanga, Verfahrenskammer, Entscheidung vom I3.6.2008. 
wird nicht in jeder Situation der richtige Weg sein. Für Europa freilich bedeutet der Nürnberger Prozess den Beginn einer lange anhaltenden friedlichen Vereinigung, die weltweit bislang ihresgleichen sucht. Das moderne Europa der Freiheit und der Rechtsstaatlichkeit hat auch in Nürnberg seinen Ursprung.

\section{Christoph Safferling}

